\title{
Gastritis varioliforme y daño hepático crónico: Una inesperada asociación
}

\author{
JUAN PABLO ROBLERO, HERNÁN ITURRIAGA, RICARDO ESTELA
}

\section{Association between varioliform gastritis and cirrhosis}

Background: Varioliform gastritis (VG) is found in approximately 0.3 to $2.8 \%$ of upper gastrointestinal endoscopies. Its etiology is not known. We have observed a higher frequency of VG in patients with liver cirrhosis. Aim: To confirm if there is an association between VG and liver cirrhosis. Patients and Methods: Two casecontrol studies were done. A retrospective study, reviewing the endoscopy database of a gastroenterological unit. A prospective study, identifying cases with the endoscopic diagnosis of VG among all patients referred for upper gastrointestinal endoscopies. The presence of liver cirrhosis, based on clinical, laboratory, ultrasonographic and endoscopic features was registered among patients with VG. Results: VG was found in 549 of 11.659 upper gastrointestinal endoscopies. Fourteen percent of patients with $V G$ had cirrhosis compared to $5.6 \%$ in control patients $\left(\chi^{2} 29,8 ; p<0.01\right)$. The odds ratio $(O R)$ for having cirrhosis of patients with $V G$ was 9.3 (95\% confidence intervals 3.4-25.5, $p<0,01$ ), according to a logistic regression analysis. In the prospective study, that included 1.498 upper gastrointestinal endoscopies, VG was also significantly more common among patients with liver cirrhosis. Conclusions: A higher frequency of VG was found among patients with liver cirrhosis. Therefore, the endoscopic finding of VG should alert physicians to look for the presence of a coexistent liver cirrhosis.

(Rev Med Chile 2010; 138: 951-956).

Key words: Endoscopy, digestive system; Gastritis; Liver cirrhosis.

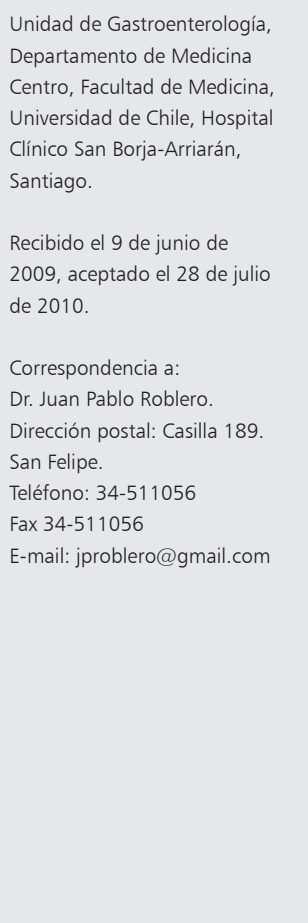

L a gastritis varioliforme (GV), también mencionada en la literatura como gastritis verrucosa o gastritis erosiva crónica, corresponde a un diagnóstico endoscópico que se basa en la presencia lesiones nodulares, habitualmente múltiples y que presentan una erosión o depresión central $^{1}$ (Figura 1).

A pesar de que su diagnóstico no es tan infrecuente, con prevalencias descritas entre 0,3 a $2,8 \%{ }^{2,3}$, es poco lo que se sabe acerca de esta entidad.

Este patrón de gastritis parece relacionarse con diversas causas y no sólo con una entidad específica. Al revisar la literatura, destaca el planteamiento de algunos grupos, quienes han manifestado que estas lesiones de la mucosa gástrica corresponderían a la expresión morfológica de una gastritis linfocítica. Esta hipótesis se ha basado en la característica histológica de las lesiones, en las que se ha encontrado un denso infiltrado linfocitario ${ }^{4-7}$; $\sin$ embargo, esto no ha sido confirmado por otros estudios ${ }^{8}$.

También, se ha evaluado su relación con el Helicobacter pylori (Hp), no encontrándose asociación evidente ${ }^{9-11}$. Asimismo, se ha descrito su presencia en pacientes con enfermedad celíaca ${ }^{11-13}$, linfoma ${ }^{9}$, enfermedad de Ménétrier ${ }^{14} \mathrm{y}$ atopia ${ }^{15,16}$.

El cuadro clínico descrito en algunos estudios es inespecífico, describiéndose principalmente epigastralgia, náuseas, anorexia, y baja de peso ${ }^{2,8}$. También, hay reportes de casos clínicos en que la manifestación principal ha sido hipoproteinemia y síndrome edematoso ${ }^{17-21}$. 


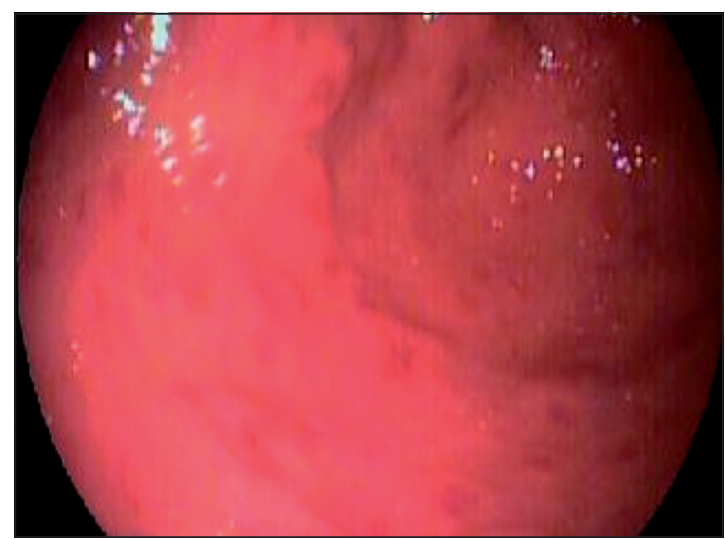

Figura 1. Gastritis varioliforme antral.

En nuestra Unidad hemos observado frecuentemente la presencia de GV en pacientes portadores de daño hepático crónico (DHC) sometidos a endoscopia digestiva alta (EDA), dato que no hemos encontrado mencionado en la literatura. Se ha descrito que en algunos pacientes cirróticos con gastropatía portal pueden observarse pliegues engrosados y de aspecto nodular, pero no la presencia de erosiones verrucosas ${ }^{22}$.

Dado esta observación, planteamos que posiblemente esta entidad es más prevalente en pacientes con DHC que en individuos sin enfermedad hepática.

El objetivo de este estudio fue evaluar esta posible asociación.

\section{Material y Método}

\section{Estudio retrospectivo}

Se realizó un estudio de casos-controles (1:2), para lo cual se revisaron las EDAs, de la base de datos de nuestra unidad, realizadas entre los años 1999 y 2004 y se seleccionaron todos los casos con diagnóstico de gastritis varioliforme o verrucosa. Se registró el diagnóstico clínico de los pacientes sometidos a EDA para confrontarlo con el diagnóstico endoscópico de GV. Se consideraron para el análisis posterior los diagnósticos clínicos de cirrosis, reflujo gastroesofágico, síndrome ulceroso, hemorragia digestiva alta, anemia y dispepsia. Se consideró como presencia de dispepsia, aquellos pacientes cuyas EDA fueron solicitadas con los diagnósticos de dispepsia propiamente tal o epigastralgia, plenitud postprandial o malestar abdo- minal alto. La presencia de cirrosis fue confirmada por la existencia de várices esofágicas (VE), además de los signos clínicos, de laboratorio y ecográficos compatibles con el diagnóstico.

También, se consideró para el análisis de los datos el diagnóstico endoscópico de várices esofágicas, esofagitis, úlcera gástrica y úlcera duodenal.

\section{Estudio prospectivo}

Se realizó igualmente un estudio de casoscontroles (1:2) incluyéndose todos los casos con diagnóstico endoscópico de GV entre octubre de 2005 y noviembre de 2006. Se registraron las mismas variables que en el estudio retrospectivo y además, en los pacientes con GV, se realizó una entrevista consultando por la presencia de comorbilidad (hipertensión arterial, diabetes mellitus, insuficiencia cardíaca, insuficiencia renal), ingesta de antiinflamatorios no esteroidales, aspirina, tabaco y alcohol. También, se tomaron 2 biopsias de las lesiones verrucosas y una biopsia de antro para estudio histológico. Para evaluar presencia de Hp se tomaron 2 muestras, una de antro y otra de cuerpo.

Estadística: Se utilizó el programa estadístico MedCalc 9.2.1.0. Se realizaron T-test, $\chi^{2}$, coeficiente de correlación, regresión logística múltiple.

El estudio fue presentado y evaluado por el Comite de Ética, reuniendo los requerimientos éticos del Comité de Bioética del Servicio de Salud del Área Metropolitana Central.

\section{Resultados}

\section{Estudio retrospectivo}

Se revisó la base de datos de los procedimientos endoscópicos entre mayo de 1997 y febrero del año 2004, la cuál contenía 14.227 endoscopias digestivas altas. Se excluyeron los pacientes con cáncer gástrico, linfoma e infección por VIH. Asimismo, en aquellos pacientes que presentaban más de un examen durante el período analizado, se consideró sólo uno de los procedimientos endoscópicos. Finalmente, se incluyeron en la revisión 11.659 endoscopias, encontrándose 549 casos con diagnóstico de gastritis varioliforme $(4,7 \%)$. Como grupo control se evaluaron 1.098 pacientes, semejantes en edad y género a los casos con GV (Tabla 1). 
El 94,4\% de las lesiones verrucosas se encontraron localizadas en el antro gástrico, 5,6\% en cuerpo y ninguna en región fúndica.

Dentro de todos los pacientes evaluados (con $y$ sin GV) se encontraron 137 pacientes con diagnóstico de cirrosis, los que fueron comparables en género y edad con los pacientes que no presentaban cirrosis (Tabla 2).

En las Tablas 1 y 3 se puede observar que el diagnóstico clínico de cirrosis fue significativamente más frecuente en el grupo con diagnóstico endoscópico de GV que en el grupo control ( $\mathrm{p}<$ 0,0001 ). En cuanto a la presencia de várices esofágicas, éstas también fueron significativamente más frecuentes en los pacientes con GV (Tabla 3). Sin embargo, al comparar la frecuencia de várices esofágicas en los pacientes cirróticos con GV y sin $\mathrm{GV}$, éstas fueron más frecuentes en el grupo de cirrosis sin gastritis varioliforme $(p=0,03)$.

Al realizar un estudio de regresión logística múltiple, se observó una correlación negativa entre GV y VE (Tabla 4).

En la Tabla 3, se observa que al correlacionar
GV con el diagnóstico clínico de solicitud de estudio endoscópico, se encuentra una asociación significativa entre GV y dispepsia $(p=0,02)$. También, resulta interesante la correlación negativa que encontramos entre GV y el diagnóstico endoscópico de esofagitis, úlcera gástrica, úlcera duodenal y anemia.

Al realizar un análisis de regresión logística múltiple, también se comprueba una fuerte asociación entre GV y DHC (OR 9,32; p < 0,0000). Se observa además, asociación significativa de GV con el diagnóstico clínico de síndrome ulceroso, dispepsia, RGE y una asociación negativa con diagnóstico endoscópico de VE, esofagitis, úlcera gástrica y úlcera duodenal (Tabla 4).

\section{Estudio prospectivo}

Durante el período de 13 meses en que se llevo a cabo este protocolo, se realizaron 1.498 endoscopias y en 58 pacientes se formuló el diagnóstico de GV (3,9\%). Como grupo control se evaluaron 116 pacientes, semejantes en edad y género a los casos con GV (Tabla 1).

Tabla 1. Características generales de pacientes de grupo retrospectivo y grupo prospectivo

\begin{tabular}{|lcccc|}
\hline & \multicolumn{2}{c}{ Grupo retrospectivo } & \multicolumn{2}{c|}{ Grupo prospectivo } \\
& Casos & Controles & Casos & Controles \\
Número & 549 & 1.098 & 58 & 116 \\
Edad (años) & $53,8 \pm 14,8$ & $54 \pm 12,1$ & $60,7 \pm 11,5$ & $63,6 \pm 12,7$ \\
Género (\%) & & & & 57,7 \\
Femenino & 66,8 & 66,3 & 56,9 & 42,3 \\
Masculino & 33,2 & 33,7 & 43,1 & 7,8 \\
\hline DHC (\%) & $13,6^{*}$ & 5,6 & $22,4^{* *}$ & 7 \\
\hline
\end{tabular}

${ }^{*} \chi^{2} 29,8 ; p=0,000 ;{ }^{* *} \chi^{2} 6,2 ; p=0,01$

Tabla 2. Características generales de pacientes con y sin cirrosis en grupo retrospectivo y grupo prospectivo

\begin{tabular}{|ccccc|}
\hline & \multicolumn{2}{c}{ Grupo } & retrospectivo & \multicolumn{2}{c|}{ Grupo prospectivo } \\
& Con DHC & Sin DHC & Con DHC & Sin DHC \\
Cirrosis (n) & 137 & 1.509 & 22 & 152 \\
Edad (años) & $53,8 \pm 12,9$ & $53,97 \pm 12,9$ & $64,2 \pm 9,8$ & $62,4 \pm 12,7$ \\
Género (\%) & & & & 59,2 \\
Femenino & 53,3 & 60,7 & 41 & 40,8 \\
Masculino & 46,7 & 39,3 & 59 & \\
\hline
\end{tabular}


Tabla 3. Coeficiente de correlación entre gastritis varioliforme y diferentes variables en grupo retrospectivo y prospectivo

\begin{tabular}{|c|c|c|c|c|}
\hline & \multicolumn{2}{|c|}{ Grupo retrospectivo } & \multicolumn{2}{|c|}{ Grupo prospectivo } \\
\hline & $\begin{array}{l}\text { Coeficiente de } \\
\text { correlación } r\end{array}$ & $\begin{array}{c}\text { Nivel de } \\
\text { significancia (p) }\end{array}$ & $\begin{array}{l}\text { Coeficiente de } \\
\text { correlación } r\end{array}$ & $\begin{array}{c}\text { Nivel de } \\
\text { significancia (p) }\end{array}$ \\
\hline Cirrosis & 0,137 & $<0,0001$ & 0,21 & 0,006 \\
\hline V. esofágicas & 0,102 & $<0,0001$ & 0,17 & 0,022 \\
\hline U. duodenal & $-0,089$ & 0,0003 & $-0,5$ & NS \\
\hline U. gástrica & $-0,059$ & 0,02 & $-0,04$ & NS \\
\hline Esofagitis & $-0,170$ & $<0,0001$ & 0,08 & NS \\
\hline Anemia & $-0,069$ & 0,005 & $-0,11$ & NS \\
\hline Dispepsia & 0,05 & 0,02 & $-0,11$ & NS \\
\hline HDA & $-0,022$ & NS & $-0,12$ & NS \\
\hline S. ulceroso & 0,005 & NS & 0,08 & NS \\
\hline RGE & 0,03 & NS & 0,06 & NS \\
\hline Edad & 0,003 & NS & $-0,11$ & NS \\
\hline Sexo & $-0,006$ & NS & 0,02 & NS \\
\hline
\end{tabular}

Tabla 4. Regresión logística múltiple entre gastritis varioliforme y diferentes variables en grupo retrospectivo

\begin{tabular}{|lcccc|}
\hline & Coeficiente & Odds Ratio & $\mathbf{9 5 \%}$ LC & p \\
\hline DHC & 2,23 & 9,32 & $3,41-25,52$ & 0,0000 \\
VE & $-1,34$ & 0,26 & $0,09-0,76$ & 0,01 \\
UG & $-1,31$ & 0,27 & $0,08-0,92$ & 0,03 \\
UD & $-1,79$ & 0,17 & $0,06-0,43$ & 0,002 \\
Esofagitis & $-1,65$ & 0,19 & $0,12-0,31$ & 0,0000 \\
Dispepsia & 0,68 & 1,98 & $1,35-2,90$ & 0,0005 \\
S. ulceroso & 0,31 & 1,36 & $1,04-1,80$ & 0,026 \\
RGE & 0,63 & 1,88 & $1,36-2,59$ & 0,0001 \\
\hline
\end{tabular}

El 100\% de las lesiones se encontraron localizadas en el antro gástrico.

Dentro de todos los pacientes evaluados se encontraron 22 pacientes con diagnóstico de cirrosis, siendo comparables en género y edad con los pacientes que no presentaban cirrosis (Tabla 2).

En la Tabla 1 y 3 se puede observar, al igual que en el grupo retrospectivo, que el diagnóstico de cirrosis, fue significativamente más frecuente en el grupo con GV que en el grupo control ( $\mathrm{p}=$ 0,01 y 0,006 respectivamente).

En Tabla 3 se aprecia que el diagnóstico endoscópico de VE en el grupo con gastritis variolifor- me, también fue significativamente más frecuente que en el grupo sin GV $(p=0,02)$. Sin embargo, no se observó diferencia al comparar la frecuencia en la prevalencia de VE en el grupo de cirrosis con GV y cirrosis sin GV.

Al realizar un estudio de regresión logística múltiple sólo se observó asociación entre GV y la variable DHC (OR 3,38; IC 1,29-8,87; p = 0,01).

Se observó un mayor consumo de aspirina y anti-inflamatorios no esteroidales en los pacientes con GV sin DHC (diferencia 27,4\%, $\chi^{2} 2,8$; $p=$ $0,09)$ y también, un mayor consumo de alcohol en los pacientes con GV y DHC (diferencia $29,5 \%, \chi^{2}$ 
$3,34 ; p=0,06)$. No hubo diferencias en comorbilidad y tabaquismo entre los pacientes con GV con DHC y GV sin DHC.

La histología en todos los casos con GV mostró un infiltrado inflamatorio leve mononuclear, pero sin predominio de linfocitos. En ninguna muestra se observó más de 30 linfocitos por campo de alta resolución, lo cual ha sido considerado como criterio diagnóstico de gastritis linfocítica ${ }^{8}$.

El test de ureasa fue positivo en $47 \%$ de los 22 casos de GV con cirrosis y en los 36 casos de GV sin cirrosis la frecuencia de test de ureasa positivo fue de $62 \%$.

\section{Discusión}

Resulta interesante destacar la alta prevalencia de GV que encontramos en nuestros pacientes, $4,7 \%$ en el grupo retrospectivo y $3,9 \%$ en el grupo prospectivo. Estas cifras son mayores que las descritas en la literatura ${ }^{1,2}$.

A través del presente estudio confirmamos la impresión que existía en nuestra Unidad sobre una mayor asociación entre GV y DHC. Se observó una relación altamente significativa tanto en el grupo retrospectivo como en el grupo prospectivo $(\mathrm{OR}=9,32$ y 3,38 respectivamente). Pensando que esta asociación podría deberse a la presencia de hipertensión portal, evaluamos la correlación entre presencia de GV y presencia o ausencia de várices esofágicas. No observamos correlación, más aún en el grupo retrospectivo encontramos una mayor asociación entre GV y DHC sin várices esofágicas. Es probable, que influya en este punto la alta prevalencia de VE de nuestros pacientes cirróticos, lo que podría explicarse por la forma en que fueron seleccionados, o sea a partir de una unidad de endoscopia. Para evaluar en forma más precisa la asociación entre GV e hipertensión portal pensamos que seria interesante evaluar un número mayor de pacientes con $\mathrm{DHC}$ sin várices esofágicas y considerar métodos más precisos en la valoración de la hipertensión portal.

Otro dato destacable, es la asociación negativa que encontramos, en el estudio retrospectivo, pero que no confirmamos en el estudio prospectivo, entre GV y úlcera gástrica, úlcera duodenal y esofagitis. En los primeros estudios de GV en la literatura, pero que se basaban en un número reducido de pacientes, se encontró asociación entre GV y úlcera duodenal ${ }^{2}$, pero posteriormente no se ha encontrado relación entre GV y enfermedad péptica ${ }^{8}$.

Se ha descrito que la GV se presenta con síntomas descritos como epigastralgia, anorexia, náuseas, baja de peso ${ }^{2,8}$.

Nosotros observamos en el grupo retrospectivo una asociación significativa con el diagnóstico clínico de síndrome ulceroso, dispepsia y RGE, planteado por el médico que solicitó la EDA. Es difícil plantear mayores conclusiones sobre esta observación, pero es posible que el síntoma epigastralgia sea predominante en estos pacientes.

En los pacientes del grupo prospectivo con GV no observamos una prevalencia mayor de infección por Hp que la observada en estudios chilenos que han evaluado la prevalencia de $\mathrm{Hp}$ en pacientes sometidos a endoscopía ${ }^{23-26}$. Esto, también concuerda con otros estudios en que no se ha observado relación ${ }^{9-11}$.

No observamos entre nuestros pacientes con GV alguno que tuviera antecedente de enfermedad celíaca o atopia, entidades que han presentado cierta asociación en algunos estudios (11-13 y 1516 respectivamente), pero es necesario considerar que no lo evaluamos dirigidamente.

Por último, nos sorprende también, la nula asociación que encontramos con gastritis linfocítica, entidad que se ha asociado a gastritis varioliforme ${ }^{4-7}$. Una posible explicación es que en los estudios en los que se ha encontrado esta asociación, las lesiones varioliformes están casi siempre localizadas en fondo y cuerpo gástrico ${ }^{3}$; por el contrario las lesiones que nosotros observamos son mayoritariamente antrales. Es probable que este patrón de gastritis corresponda a diversas etiologías y que en nuestros casos, en que la localización es antral, la patogenia esté más relacionada con otras causas que tengan alguna relación con la presencia de DHC.

Estimamos que este estudio tiene importancia en la práctica clínica habitual, en el sentido de qué el hallazgo endoscópico de gastritis varioliforme debería alertar al clínico sobre la mayor posibilidad de presencia de una enfermedad hepática crónica coexistente.

En este estudio, no encontramos asociación con presencia de várices esofágicas, pero sería interesante evaluar las alteraciones microcirculatorias y las alteraciones en los mecanismos de defensa de la mucosa gástrica presentes en los pacientes cirróticos que podrían predisponer a este tipo de injuria de la mucosa. 


\section{Referencias}

1. Green PH, Gold RP, Marboe CC, Weinberg LM, Goldfarb JP, Brasitus TA. Chronic erosive gastritis: clinical, diagnostic, and pathological features in nine patients. Am J Gastroenterol 1982; 77: 543-7.

2. Green PH, Fevre DI, Barrett PJ, Hunt JH, Gillespie PE, Nagy GS. Chronic erosive (verrucous) gastritis. A study of 108 patients. Endoscopy 1977; 9: 74-8.

3. Lambert R, André C, Moulinier B, Bugnon B. Diffuse varioliform gastritis. Digestion 1978; 17: 159-67.

4. Haot J, Hamichi L, Wallez L, Mainguet P. Lymphocytic gastritis: a newly described entity: a retrospective endoscopic and histological study. Gut 1988; 29: 1258-64.

5. Haot J, Berger F, Andre C, Moulinier B, Mainguet P, Lambert R. Lymphocytic gastritis versus varioliform gastritis. A historical series revisited. J Pathol 1989; 158: 19-22.

6. Haot J, Jouret A, Willette M, Gossuin , Mainguet P. Lymphocytic gastritis: prospective study of its relationship with varioliform gastritis. Gut 1990; 31: 282-5.

7. Rutgeerts L, Stuer A, Vandenborre K, Guillebert G, Tanghe W. Lymphocytic gastritis: Clinical and endoscopic presentation and long-term follow-up. Acta Gastroenterol Belg 1995; 58: 238-42.

8. Ribeiro VL, Barbosa AJ, Barros CA. Varioliform gastritis: frequency and relationship whith lymphocytic gastritis. Arq Gastroenterol 2000; 37: 7-12.

9. Miettinen A, Karttunen TJ, Alavaikko M. Lymphocytic gastritis and Helicobacter pylori infection in gastric lymphoma. Gut 1995; 37: 471-6.

10. Niemela S, Karttunen T, Kerola T, Karttunen R. Ten year follow up study of lymphocytic gastritis: further evidence on Helicobacter pylori as a cause of lymphocytic gastritis and corpus gastritis. J Clin Pathol 1995; 48: 1111-6.

11. Wu TT, Hamilton SR. Lymphocytic gastritis: association with etiology and topology. Am J Surg Pathol 1999; 23: 153-8.

12. De Giacomo C, Gianatti A, Negrini R, Perotti P, Bawa P, Maggiore G, Fiocca R. Lymphocitic gastritis: a positive relationship with celiac disease. J Pediatr 1994; 124: 57 62.

13. Feeley KM, Heneghan MA, Stevens FM, McCarthy CF. Lymphocytic gastritis and coeliac disease: evidence of a positive association. J Clin Pathol 1998; 51: 207-10.

14. Haot J, Bogomoletz WV, Jouret A, Mainguet P. Ménétrier disease with lymphocytic gastritis : an unusual association with possible pathogenic implications. Hum Pathol
1991; 22: 379-86.

15. Andre C, Moulinier B, Andre F, Daniere S. Evidence for anaphylactic reactios in peptic ulcer and varioliform gastritis. Ann Allergy 1983; 51: 325-8.

16. Andre C, Guillon J, Moulinier B, Martin A, Fargier MC. Randomised placebo-controlled double blind trial of two dosages of sodium cromogycate in treatment of varioliform gastritis: comparison with cimetidine. Gut 1982; 23: 348-52.

17. Farahat K, Hainaut P, Jamar F, Lambert M. Lymphocytic gastritis: an unusual cause of hypoproteinaemia. J Intern Med 1993; 234: 95-100.

18. Murata I, Yoshikawa I, Kuroda T, Tabaru A, Miura T, Otsuki M. Varioliform gastritis and duodenitis associated with protein-losing gastroenterpathy, treated with omeprazole. J Gastroenterol 1996; 31: 109-13.

19. Cacoub P, Sbaï A, Costedoat-Chalumeau N, Bellanger J, Godeau P, Piette JC. Edematous syndrome revealing an exudative lymphocytic gastritis: efficacy of omeprazole. Gastroenterol Clin Biol 2000; 10: 963-4.

20. Tissot B, Tropet AL, Blanchi A, Visée S. Lymphocytic gastritis with anasarca and venous thrombosis. Presse Med 2006; 35: 974-6.

21. Montagnac R, Blaison D, Ciupea A, Für A, Pradel J, Scillinger F. A rare cause of peripheral edema: exudative lymphocytic gastritis induced hypoprotidemia. Nephrol Ther 2007; 3: 107-12.

22. Chang D, Levine MS, Ginsberg GG, Rubesin SE, Laufer I. Portal hypertensive gastropathy: radiographic findings in eight patients. AJR Am J Roentgenol 2000; 175: 160912.

23. Csendes A, Smok G, Burdiles P, Blanco C, Rodas J. Presencia de Helicobacter pylori en el duodeno, antro $\mathrm{y}$ fundo de sujetos controles y pacientes con úlcera duodenal, úlcera gástrica, gastritis o duodenitis erosiva. Análisis histológico de 357 sujetos. Rev Med Chile 1995; 123: 177-84.

24. Giglio M, Hoffenberg P, Becker L, Ossa P, Dueñas J, Reyes V, et al. Campylobacter pilorides: Prevalencia en endoscopías normales, gastritis atrófica y úlcera péptica. Rev Med Chile 1988; 116: 229-31.

25. Prado V, Wenger J, Klinger J, Latorre J, Maggiolo P, Martínez J et al. Campylobacter pylori en niños y adultos sometidos a endoscopía: Correlación de hallazgos endoscópicos, bacteriológicos e histologías. Rev Med Chile 1989; 116: 503-8.

26. Figueroa G, Acuña R, Troncoso M, Portell DP, Toledo MS, Valenzuela J. Helicobacter pylori infection in Chile. Clin Infect Dis 1997; 25: 983-9. 